\title{
Soft X-ray Spectrotomographic Microscopy at Cryogenic Temperatures
}

J. Geilhufe $^{1}$, A. F. G. Leontowich ${ }^{1}$, J. Wang ${ }^{1}$, R. Berg ${ }^{1}$, C. N. Regier ${ }^{1}$, D. M. Taylor ${ }^{1}$, D. Beauregard ${ }^{1}$, J. Swirsky ${ }^{1}$, C. Karunakaran ${ }^{1}$, A. P. Hitchcock ${ }^{2}$ and S. G. Urquhart ${ }^{3}$

1. Canadian Light Source Inc., Saskatoon, SK S7N 2V3, Canada

2. Brockhouse Institute for Materials Research, McMaster University, Hamilton, ON L8S 4M1, Canada

3. Department of Chemistry, University of Saskatchewan, Saskatoon, SK S7N 5C9, Canada

* Corresponding author, jan.geilhufe@lightsource.ca

Soft X-ray spectromicroscopy is a well-established and powerful technique that has led to significant advancements in diverse fields such as material science, medicine, nanofabrication, as well as agriculture and environmental research [1]. The method relies on recording a series of high-resolution, monochromatic images over a photon energy range of interest in order to obtain spatially resolved X-ray absorption spectra (XANES) [2]. Modern soft X-ray scanning transmission X-ray microscopes (STXM), such as the ambient-STXM at the Canadian Light Source (CLS), acquire images with a spatial resolution of $30 \mathrm{~nm}$ (half pitch) for sub-micrometer thick specimens [3]. A conversion of the spatially resolved XANES spectra into quantitative chemical maps is routinely accomplished using reference spectra [4]. Depending on desired spectral resolution and spectral/chemical complexity, such measurements may necessitate a stack of up to 100 images, so that methods to limit radiation damage are crucial for studies of highly radiation sensitive materials such as polymers and biological samples. In a STXM, the Fresnel zone plate (FZP) diffraction order is selected upstream of the specimen and the radiation dose deposited on the sample is thus limited to the essential X-rays, whereas the inefficient optic is located after the sample in full field transmission microscopes (TXM). In addition to 2-dimensional analysis of thin samples, the extraction of chemical information from volumetric specimens via tomography at multiple photon energies has become increasingly popular [5]. For a spectrotomographic dataset, the region-ofinterest needs to be scanned multiple times, not only over different energies, but also over a series of tilt angles, and/or polarization states. Thus, the accumulated dose deposited in the sample increases significantly in spectrotomography. Several approaches to limit dose include: minimizing the energies to those essential for chemical analysis; and the use of new tomographic reconstruction algorithms, such as compressed sensing [6], that need of relatively few tilt angles for acceptable results. Despite these approaches, radiation damage is a significant challenge to 4D imaging, especially for extremely radiation sensitive samples such as the ionomer in polymer electrolyte membrane fuel cell (PEM-FC) devices [7].

It has been shown that the radiation robustness of the specimen can be significantly increased by cryogenic cooling [8]. At the CLS, we are currently commissioning a new cryoSTXM (Fig. 1a,b) optimized for tomographic measurements at the Spectro-Microscopy (SM) beamline (130-2700 eV). The new CLS cryo-STXM offers fast-scanning and semi-automated spectrotomographic measurements with cryogenic sample cooling to limit mass loss radiation damage. The cryo-STXM works in near ultra-high vacuum to minimize sample contamination by condensed water or organics. It consists of a high-tilt tomography cryotransfer system adapted from cryo-tomography instruments used in transmission electron microscopy. The sample can be positioned with tilt angles from $-70^{\circ}$ to $+70^{\circ}$ while maintaining stable sample temperature between $-180^{\circ} \mathrm{C}$ and $+90^{\circ} \mathrm{C}$. A differentially pumped load-lock affords fast sample exchange. Images are acquired by scanning the FZP with the sample stationary. Along with the new STXM, a new control and acquisition system, pySTXM, has been developed, which is designed to optimally support the experimenter through all stages of the measurement, starting from the initial calibrations of the components to the beam axis all the way through automated measurements of image 
stacks or multi-tilt angle spectrotomographic measurements.

In order to further increase the beamline efficiency, we recently installed a laser system that allows for imaging with a focused blue laser, along the same beam path as the X-rays. This system is being used to develop the cryo-STXM when X-rays are not available. This has helped to tune the scanning stages for significantly increased performance while minimizing distortions. More optimization potential can be unlocked by using the laser system prior to scheduled X-ray beamtimes to characterize the sample, to identify suitable ROIs for the X-ray measurements, and to adjust the cryotomo goniometer to establish eucentric conditions. The optical scan mode is a valuable and damage-free measuring mode that improves overall beamline efficiency and helps to minimize X-ray exposure for sensitive samples.

Fig.1c is an example of a spectrotomography measurement carried out during commissioning of the CLS cryo-STXM. It displays the 3D spatial distribution of perfluorosulfonic acid ionomer (red) relative to catalyst support (blue) in a microtomed section of the cathode of a PEM-FC. Pre- and postacquisition $\mathrm{F}$ 1s spectra of this region were identical, which had not previously been possible with room temperature spectrotomography of the same material [7]. The CLS cryo-STXM will soon be available for to the international user community, for both per-review and fee-for-service access [9].
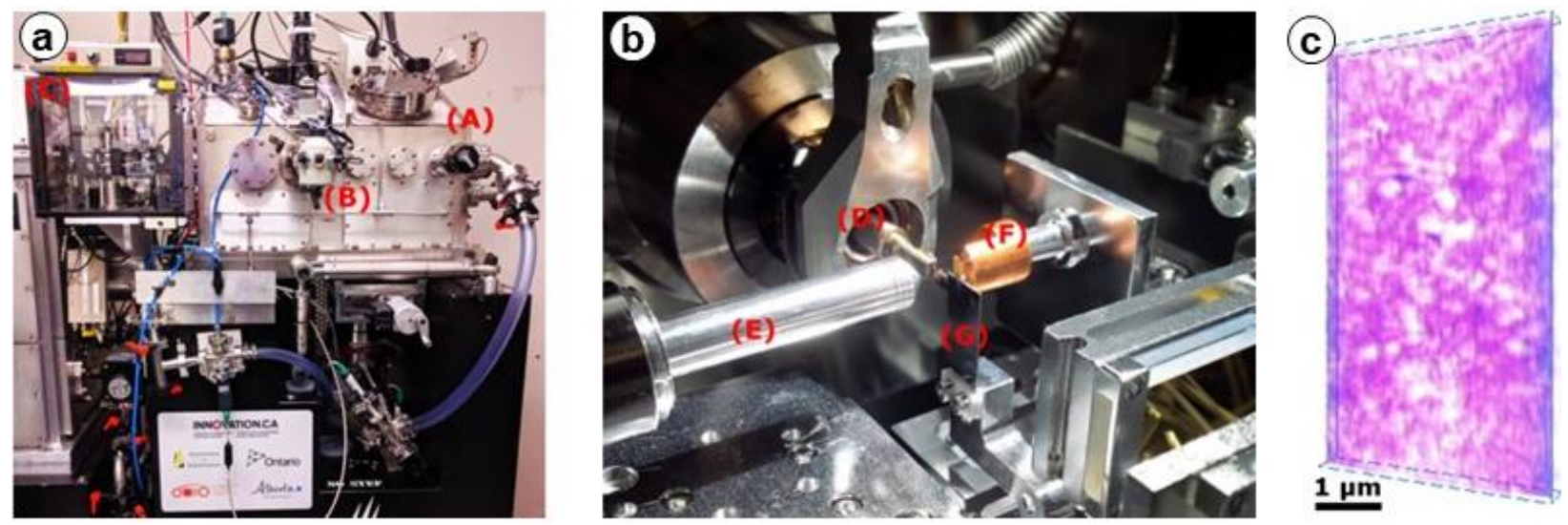

Figure. 1. (a) The CLS cryo-STXM at the SM beamline. (A) Vacuum tank with vacuum infrastructure, (B) High-tilt tomography cryotransfer system. (C) $405 \mathrm{~nm}$ laser system for optical sample scanning. (b) Inside view of the cryo-STXM. (D) High-tilt tomography cryotransfer system with sample. (E) Point detector (PMT). (F) Scanning FZP with optimized mounting for tomographic imaging. (G) Order sorting aperture. (c) 3D spatial distribution of perfluorosulfonic acid ionomer (red) relative to catalyst support (blue) in a microtomed section of the cathode of a PEM-FC.

\section{References}

[1] CLS-SM publications: https://sm.lightsource.ca/publications/

[2] C. Jacobsen, et al, J. Microsc. 197, 173-184 (2000).

[3] K.V. Kaznatcheev, et al, Nucl. Inst. Meth. Phys. Res., Sect. A 582, 96-99 (2007).

[4] H. Ade and A. P. Hitchcock, Polymer 49, 643-675 (2008).

[5] G. Schmid in "X-ray and Neutron Techniques for Nanomaterials Characterization", 5, C.S.S.R. Kumar, ed. 43-94 (Springer, Berlin, 2016)

[6] J. Wu et al. Microscopy \& Microanalysis 23, 951-956 (2017).

[7] J. Wu et al. J. Power Sources 381, 72-83 (2018).

[8] T. Beetz and C. Jacobsen, J. Synchrotron Rad. 10, 280-283 (2003).

[9] We acknowledge the funding partners of CLS. Cryo-STXM funded by CFI, AFCC \& provinces of Alberta, Ontario and Saskatchewan. 